\title{
The Determination of Bile Acids in Women Upon Suspicion of Intrahepatic Cholestasis of Pregnancy
}

\author{
Miroslava Beňovská ${ }^{1,2}$, Jana Tomanová ${ }^{1}$, Michaela Červienková ${ }^{2}$, Petr Janků ${ }^{3}$ Patrícia Serpa Nižnik ${ }^{3}$, Martina \\ Podborská ${ }^{1}$ and Ondřej Wiewiorka ${ }^{1,2}$ \\ ${ }^{1}$ Department of Clinical Biochemistry, University Hospital Brno, Czech Republic \\ ${ }^{2}$ Department of Laboratory Methods, Faculty of Medicine, Masaryk University, Czech Republic \\ ${ }^{3}$ Department of Gynecology and Obstetrics, University Hospital Brno and Faculty of Medicine, Masaryk University, Czech Republic
}

Submission: May 11, 2021; Published: May 21, 2021

*Corresponding author: Miroslava Beňovská, Department of Clinical Biochemistry, University Hospital Brno, Jihlavská 20, 62500 Brno, Czech Republic

\begin{abstract}
Background: Bile acids are used in testing of women with suspicion of intrahepatic cholestasis of pregnancy, but the influence of their levels on diagnoses, week of delivery and response to treatment remains unclear.

Methods: We retrospectively evaluated the serum levels of total bile acids in 217 singleton pregnant women measured by photometric enzymatic method from Dialab.

Results: Medians of bile acids in serum of women with intrahepatic cholestasis of pregnancy increased compared to normal pregnancies (23.4 (19.5-28.2) versus 3.7 (3.0-4.2); $\mathrm{P}<0.0001)$. ROC analysis for bile acids showed AUC of 0.942 ( $95 \%$ CI, 0.902-0.969), $\mathrm{P}<0.0001$, a sensitivity of $85,9 \%$ and a specificity of $94,9 \%$ for intrahepatic cholestasis of pregnancy. The statistically significant correlation between levels of bile acids and ALT, AST and ALP was proved. The difference in week of delivery between group with bile acid $<40 \mu \mathrm{mol} / \mathrm{L}$ and $>40 \mu \mathrm{mol} / \mathrm{L}$ was about 1 week $(\mathrm{P}=0.01)$. The dynamics of concentration of bile acids after treatment by hepatoprotectives was monitored as well. We observed mostly either decreased or unchanged concentration of bile acids, but in almost $24 \%$ of cases, there was a rapid and significant increase of bile acids concentration, despite the treatment.
\end{abstract}

Conclusion: The presented results show that bile acids represent useful parameter, which can detect intrahepatic cholestasis of pregnancy.

Keywords: Intrahepatic cholestasis of pregnancy; Bile acids; Hepatoprotectives; Stillbirth; Pruritus

\section{Introduction}

The determination of bile acids in women upon suspicion on intrahepatic cholestasis of pregnancy (ICP) is substantially important and it is gaining on significance these days [1]. ICP is a reverse form of cholestasis occurring in pregnancy with symptoms of dysfunction of liver [2]. It is the most common primary liver disorder in pregnant women, affecting about $0.5-1 \%$ of pregnancies [3-5] and associated with numerous adverse perinatal outcomes [6]. The fetus is threatened by arrhythmia or cardiac arrest and is endangered by the aspiration of meconium-stained amniotic fluid. This fetal distress may lead to premature birth $[7,8]$. Stillbirth complicates $0.83 \%$ of ICP. While the risk of stillbirth is increased in women with ICP, it is significantly greater than general

population rates only when the concentration of serum bile acids exceeds $100 \mu \mathrm{mol} / \mathrm{L}$ [6]. ICP is characterized by pruritus without any skin lesion that occurs in the late second to third trimester of pregnancy [9-11] and raised concentrations of maternal bile acid $[2,3,9]$. Usually, elevated liver transaminases are present $[1,3,12]$; 2-10 times higher levels were observed in about $60 \%$ of patients with increased bile acids [12]. Hyperbilirubinemia is not present [11]. ICP has a multifactorial etiology [3,5]. The genetic influence is supported by increased rate of ICP found in some families and by difference in prevalence between ethnic groups. The main genes responsible are linked to bile acid excretion. Endocrine factors are involved as well because of increased serum levels of sulphonated 


\section{Global Journal of Reproductive Medicine}

progesterone metabolites found in women with ICP mainly in the third trimester and are linked to disease severity [13]. Also, women with a history of allergic reactions were more likely to develop intrahepatic cholestasis of pregnancy [4]. Populationbased studies have highlighted environmental factors contributing to the disease, including reduced dietary selenium, low vitamin D and winter months $[3,13]$. This is supported by higher incidence of ICP $(2.7 \%)$ in Iceland compared to the rest of the Europe $(0.5 \%)$ [3].Upon suspicion of ICP, it is necessary to exclude viral and autoimmune causes of raised bile acids andamino transaminases $[5,14]$. However, it was showed that elevated hepatitis $C$ viral load does not influence bile acids level and the fetal left ventricular Tei index [15]. Increased transport of bile acids from mother with ICP to fetus is proved by elevated level of bile acids in amniotic fluid, umbilical vein, and meconium. Cholic and chenodeoxycholic acids pass through placenta and have toxic influence on the fetus and placenta as well [9]. There is a positive correlation between the concentration of bile acids in the umbilical vein $[16,17]$ and the damage of placenta in ICP [16]. According to the literature, ICP could be divided into an early-onset $(<33-34$ gestational week) and a late-onset ( $\geq 33-34$ gestational week) $[18,19]$. Adverse pregnancy or fetal outcomes and preterm birth affect significantly more patients with the early-onset ICP [18]. Another criterion of how to divide women with ICP is into mild, moderate, and severe groups (bile acids concentration 10-39, 40-99 and $>100 \mu \mathrm{mol} / \mathrm{L}$ resp.). It has been published that the gestational age at diagnosis and at delivery were significantly lower in the severe ICP group, as compared with the mild one.Also, meconium-stained fluid (47.6\%), and perinatal death (9.5\%) occurred significantly more often in cases with severe ICP [17]. Contemporarily, the common treatment of ICP is a dosage of a naturally hydrophilic bile acid ursodeoxycholic acid (UDCA), which leads to a decreased amount of bile acids and a reduction in total preterm births [20,21]. The possible effect of UDCA includes improved bile acids transportation and detoxification, cyto protection, and antiapoptotic effect [22]. It was also reported that UDCA stimulates hepatobiliary secretion of bile acids and prevents cholestasis induced by hydrophobic bile acids [23]. The bile acids concentration had been quickly lowered under $10 \mu \mathrm{mol} / \mathrm{l}$ in treated women, but their liver function and bile acid profiles were still not back to normal [20]. Not all pregnant women with pruritus are diagnosed with ICP. Part of them has no liver damage and no elevated bile acids till the delivery. The pruritus in these cases is a benign condition without complications for the mother and fetus, called benign pruritus gravidarum $[4,24,25]$. As the level of bile acids changes during pregnancy, it must be considered, that part of those women later develop abnormal bile acids results, and their diagnosis is changed to ICP [4]. On the other hand, even with increased serum bile acid levels, the pruritus is not sometimes present. Feng's study recorded that asymptomatic pregnant women with increased serum bile acid levels at least two times during pregnancy were at similar or higher risk of stillbirth than patients with typical ICP [26]. The most appropriate biologic material for the determination of bile acids is fasting serum as was found in a study comparing results after fasting and glucose load with an approximate $2 \mu \mathrm{mol} / \mathrm{L}$ difference [27].A frequent method for the analysis of total bile acids is enzymatic photometry with thio-NAD or nitro tetrazolium blue chromophore. POCT bile acids biosensors are also developed and described in the literature. To gain the profile of individual bile acids, HPLC, HPLC-MS, GC-MS [1] or capillary electrophoresis [28] was used. By HPLC MS/MS spectroscopy, it was discovered that the unconjugated bile acids were decreased in women with ICP and taurin conjugates and glycin conjugate bile acids were increased (taurocholic acid, tauro- $\alpha$-muricholic acid and tauro$\omega$-muricholic acid) [20]. Similar results have been reached and described in other study [29]. It was also published that lithocholic acid and ursodeoxycholic acid/ lithocholic acid ratio could provide better and more accurate information for the diagnosis of ICP than total bile acids levels [28]. The aim of this retrospective study was to statistically evaluate the influence of bile acids concentrations on diagnoses and week of delivery. Response to treatment in a group of pregnant women with suspected ICP was described.

\section{Materials and Methods}

In this retrospective study, serum levels of bile acids in a group of 217 singleton pregnant women with suspicion to ICP were analyzed. The samples were collected at the Department of Gynecology and Obstetrics from July 2016 to April 2019 and measured at the Department of Clinical Biochemistry. For illustration, in the same period, the total of 18370 deliveries were performed in the hospital. The diagnosis of the ICP was confirmed by a gynecologist according to the typical clinical symptoms and pathology in biochemical parameters (increased bile acids, AST, ALT, ALP).The bile acids analyses were performed repeatedly over the course of patient's treatment, so only the sample with the highest bile acids concentration was selected for the first part of statistical evaluation. The second part of statistical evaluation studying the hepatoprotective treatment effect utilized data from all patient's samples.Out of the 217 observed pregnant women, there were 99 cases of women with ICP, while the group with other diagnosis counted 118 cases. This second group included women with benign pruritus gravidarum (PG), women with ICP or other problems in previous gravidity, individuals with preeclampsia or abnormal hepatic function test results due to other causes. The bile acids determination in serum samples was carried out on clinical chemistry module c502 of cobas 8000 (F.Hoffman-La Roche Ltd., Basel, Switzerland; further as Roche) by enzymatic photometric assay Bile Acids from Dialab (Dialab Produkten und Laborinstrumenten Gesellshaft m.b.H, Wiener Neudorf, Austria). The principle of the method is based on photometric cyclic enzymatic reaction of bile acids with Thio-NAD in presence of enzyme 3- $\alpha$-hydroxysteroid dehydrogenase (3- $\alpha$-HSD) [30]. Reference range according to the reagent's manufacturer is 0 $10[\mu \mathrm{mol} / \mathrm{L}]$. Further details about methodologies are given in Dialab insert for the test [30]. Statistical analysis were performed with a statistical software Medcalc (MedCalc Software, Ostend, Belgium), version 9.3.2.0. The data in groups of pregnant women with and without ICP were compared by Mann-Whitney tests 


\section{Global Journal of Reproductive Medicine}

and a receiver operating characteristic (ROC). The correlation among bile acids and ALT, AST respectively ALP were calculated by Spearman's coefficients (202 women), as normality of the values distribution was rejected by D'Agostino-Pearson test. The Spearman's coefficients were calculated only for the group of 202 women as in some of the cases the ALT, AST and ALP concentrations were not known (patients from other health care facilities who were measured for bile acids only in our hospital). All $\mathrm{P}$ values were two-tailed. An independent t-test was used to inspect a possible difference in week of delivery in dependence on bile acid concentration. A total of 87 women (as 13 women did not give birth in our hospital, so their data could not be used) were divided into two groups, first with bile acids $\leq 40 \mu \mathrm{mol} / \mathrm{L}$ and second with bile acids $>40 \mu \mathrm{mol} / \mathrm{L}$ [31].Normality of data was verified by a D'Agostino-Pearson test and arithmetic means and medians of both groups were established. The course and effect of treatment by hepatoprotectives such as Essentiale, Urosan and Transmetyl, in different combinations, was monitored on a group of 35 pregnant women in which the bile acids were measured repeatedly during pregnancy. The level of bile acids was analyzed 2-11x within this group and results were divided into four groups according to reaction to the treatment. The study was performed in accordance with the Declaration of Helsinki, institutional policies and has been approved by the local Ethical Committee -
No. 02-090119/EK.

\section{Result}

Medians and confidence intervals of bile acids for group of pregnant women with and without ICP are given in (Table 1).The median of serum concentration of women with ICP were significantly higher than that of the women with other diagnosis. In the (Figure 1\&2), there can be seen different effect of hepatoprotectives as Essentiale, Urosan and Transmetyl on individuals with ICP. The total of 35 monitored individuals was divided into 4 groups. In group A the decrease of bile acids concentration was observed since the treatment started. In some cases, bile acid concentration increased in a few weeks again despite the treatment. In group B patients got hepatoprotectives due to increased values of ALT and AST or/and pruritus only. At this moment bile acids were in reference range and slightly increased later. In group $\mathrm{C}$ we have seen an increase of bile acids concentration, often dramatic in course of 1-3 weeks of therapy. Group D had individuals whose concentration stayed even (with pathologic values) for few weeks, in some cases followed by a rather small increase. Out of 35 women, only 27 had pruritus at least in some week of pregnancy. Three of them had meconiumstained amniotic fluid (one of groups A, B and C).

Table 1: Comparison of a group of women with ICP and a group of other pregnant women.

\begin{tabular}{|c|c|c|c|}
\hline \multirow{2}{*}{ Concentration $[\mu \mathrm{mol} / \mathrm{L}]$} & \multicolumn{2}{|c|}{ Median and $95 \%$ Interval of Confidence } & \multirow{2}{*}{$\mathbf{P}$} \\
\hline & Women with ICP $(\mathrm{N}=99)$ & Others $(\mathrm{N}=118)$ & \\
\hline Bile acids & $23.4(19.5-28.2)$ & $3.7(3.0-4.2)$ & $<0.0001$ \\
\hline
\end{tabular}

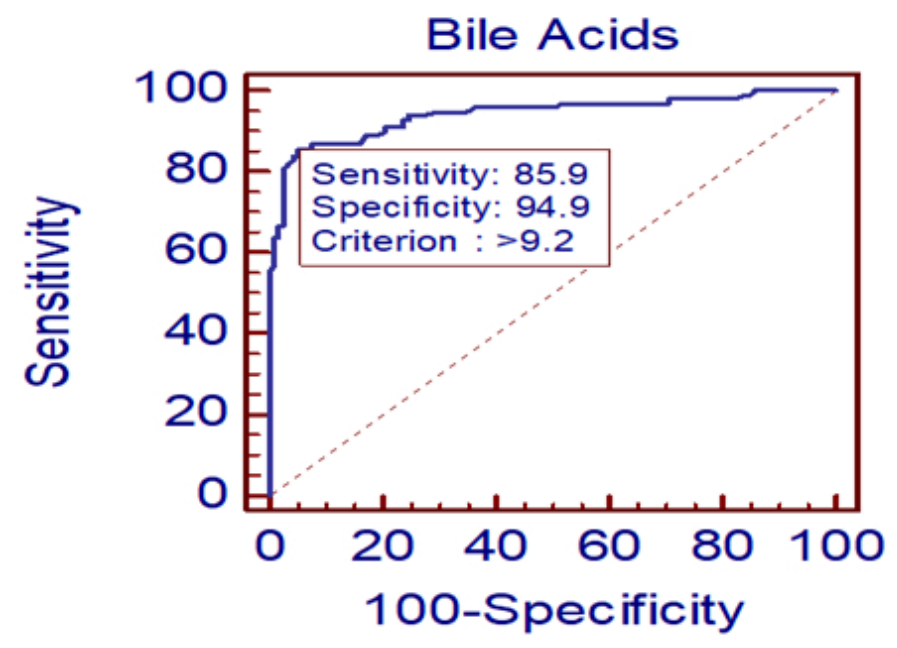

Figure 1: ROC curve for the bile acids discriminating women with ICP and with other diagnosis.

Group of pregnant women - 99 with ICP, 118 others: AUC = 0.942, $P<0.0001$. 


\section{Individuals according to bile acids dynamics after treatment}

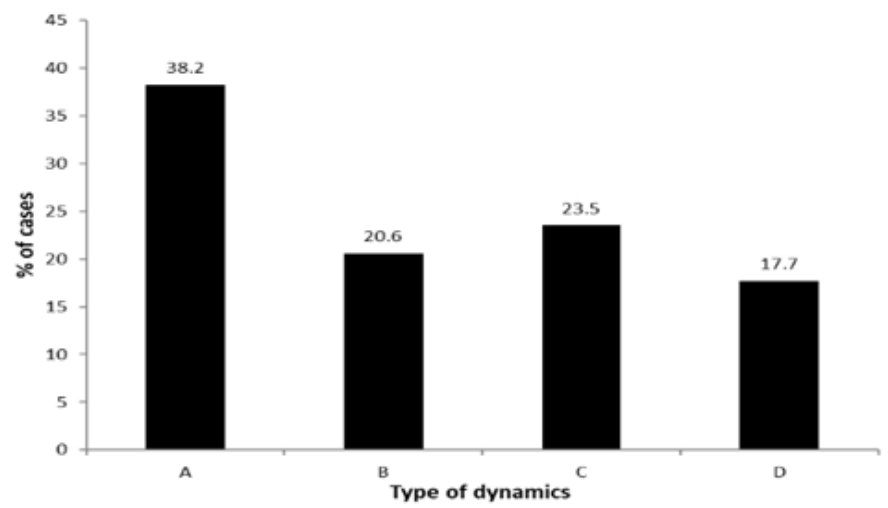

Figure 2: The division of individuals according to dynamics of concentration of bile acids after beginning of treatment by hepatoprotectives. A: Decrease.

B: Small increase from normal values.

C: Increase (often dramatic) in 1-3 week.

D: Even for few weeks.

Table 2: Correlation between levels of bile acids and others biochemical parameters (202 individuals).

\begin{tabular}{|c|c|c|}
\hline Parametr & Spearman's Coefficient of Rank Correlation (rho) & Significance Level \\
\hline ALT & $0.674(95 \% \mathrm{CI}, 0.591-0.743)$ & $\mathrm{P}<0.0001$ \\
\hline AST & $0.696(95 \% \mathrm{CI}, 0.618-0.761)$ & $\mathrm{P}<0.0001$ \\
\hline ALP & $0.575(95 \% \mathrm{CI}, 0.474-0.660)$ & $\mathrm{P}<0.0001$ \\
\hline
\end{tabular}

\section{Discussion}

Whereas the determination of bile acids for pregnant women with suspicion to ICP (individuals with pruritus or pathological transaminases) is relatively commonly used in practice, the statistical evaluation as presented in this article was not, according to our knowledge, yet presented.The Mann-Whitney test points to a great difference of means of bile acids concentration between the group of women with ICP $(23.4 \mu \mathrm{mol} / \mathrm{L})$ and the group of women with other diagnosis $(3.7 \mu \mathrm{mol} / \mathrm{L})$ showing statistical significance with $\mathrm{P}<0.0001$. The ROC analysis showed steep curve with excellent value of AUC (0.942). The counted ideal associated criterion (limit value between negative and positive outcome of the test) of $9.2 \mu \mathrm{mol} / \mathrm{L}$ is quite close to reference range mentioned in the method insert $(0-10 \mu \mathrm{mol} / \mathrm{L})$ [30]. We suspect that the results could be even better as some patients were already getting hepatoprotectives from their gynecologists right after reaching increased transaminases values and/or pruritus, before they arrived at our hospital. In these cases, the bile acids were measured at the hospital at a time when they could already be decreased. In group of pregnant women with other diagnosis, the individuals with pruritus but no liver damage and no elevated bile acids were observed till the delivery. In one case the pruritus was caused by dermatitis, other cases fill the described condition called benign pruritus gravidarum [4,24].A fair correlation between bile acids concentrations and ALT, AST and ALP levels was found - see (Table $2 \& 3$ ). The best correlation of bile acids was with AST - Spearman's coefficient of 0.696. Results show that the bile acids are better than ALT, AST, and ALP for the diagnosis of ICP.The disadvantage of using ALT and AST for diagnosis determination of ICP is that the transaminases can be elevated also by other reasons than ICP as preeclampsia, chronic hepatitis, autoimmune hepatopathy. According to literature [12], 2-10 times higher levels of ALT, AST were observed in about $60 \%$ of patients with increased bile acids. In our study, all women with ICP had increased transaminases; only $10 \%$ of them less than twice the upper value of their respective reference ranges.The ALP values are physiologically elevated in II. and III. trimester due to the production of placental isoenzyme and it is generally considered to be of no great diagnostic importance to ICP [12]. On the other hand, compared to normal pregnancy, the ALP values are even more elevated in the case of ICP according to guideline of national gynecological and obstetric society (CGOS) and our observations. The difference in the week of delivery between group with bile acid to $40 \mu \mathrm{mol} / \mathrm{L}$ and group with bile acids $>40 \mu \mathrm{mol} / \mathrm{L}$, which is about 1 week, is relatively small, but significant. Results are influenced by ICP treatment and by the fact that, in accordance with CGOS guideline, most deliveries are induced after the end of 37 week of gestation in women with ICP even if the treatment is successful. It corresponds to UK practice, as most cases there undergo induction of labor at week $37-38$ as this is considered 


\section{Global Journal of Reproductive Medicine}

to balance the effects of prematurity against risk of fetal demise, which is greatest between 37 and 39 weeks of gestation [9]. It is generally known that after a beginning of hepatoprotective treatment, in some cases bile acids levels continue to increase. In this study we observed mostly either decreased or unchanged concentration of bile acids, but in almost $24 \%$ of cases, there was a rapid and significant increase of bile acids concentration at the next measurement, despite the treatment. Among others, in this group there was one case of meconium-stained amniotic fluid and one case of silent fetus outcome (this was the only case in all tested pathologic group of 217 with an outcome of silent fetus despite treatment, monitoring and decrease of bile acids value to $22 \mu \mathrm{mol} / \mathrm{L}$ ). Individual groups are too small to make a conclusion, but the described possible reactions to treatment should be considered. Responses to the treatment may be affected by the severity of ICP, a week of pregnancy when the disease occurs and by the combination of hepatoprotectives. This implies that monitoring of bile acids levels is very important even after a treatment is applied. In some cases, the considerable decrease of bile acids and even transaminases values were observed in 1-3 weeks of the treatment, where the concentration could reach normal values. But a decrease of ALT and AST values usually steadied at values of $1-2 \mu \mathrm{kat} / \mathrm{L}$. Although the hepatoprotectives has a great significance, the decrease or even return of bile acids values to normal does not mean that the women are without risk, based on both literature [20] and our observations. Values of bile acids are also not $100 \%$ specific, as they are increased in patients with cholecystolithiasis or cholestasis, but in the observed group of 227 individuals was only 1 with increase bile acids from other reason than ICP. The cause was cholecystolithiasis, a rare diagnosis in pregnant women. Within the group with other diagnosis than ICP, individuals with only preeclampsia had normal bile acids. We found 4 cases within the ICP group, where preeclampsia and ICP was combined. As mentioned in the chapter Materials and methods, twin pregnancy were not included to the study, although according to our observation, in healthy pregnancy the levels of bile acids stay in normal values, independently on the number of fetuses. Unfortunately, we had not enough cases to prove this claim which can be a subject for further study.Another topic that would be interesting to further investigate in the future are normal values of bile acids in umbilical cord. In the literature, there is only information about positive correlation between levels of bile acids in serum and the umbilical vein and finding that values in umbilical cord blood are significantly lower [15].Exact reference ranges for umbilical cord blood are not published according to our knowledge yet. In conclusion bile acids are very good and useful diagnostic parameters for ICP. They are superior to the less specific ALT, AST, and pruritus presence as well. Although results of analysis of individual bile acids in literature $[26,28,29]$ clarify the mechanism of ICP in the important and interesting way, according to our experiences, total bile acids measurement is sufficient in routine practice and correlates well with the patient's condition. Our results supported bile acids importance and uniqueness.

Table 3: Difference in medians and means of week of delivery between two groups of women in dependence on their bile acids concentrations (treshold value $=40 \mu \mathrm{mol} / \mathrm{L}$ )

\begin{tabular}{|c|c|c|c|}
\hline $\mathbf{N}$ & Concentration $[\mu \mathrm{mol} / \mathrm{L}]$ & \multicolumn{2}{|c|}{ Week of Gravidity at Delivery } \\
\hline \multicolumn{2}{|c|}{ Bile Acids } & Median & Arithmetic Mean \\
\hline 59 & $\leq 40$ & $38+1(95 \%$ CI, 37+4 - 38+6) & $38+1(95 \%$ CI, $37+4-38+4)$ \\
\hline 28 & $>40$ & $37+2(95 \%$ CI, 36+4 - 37+6) & $36+7(95 \% \mathrm{CI}, 36+2-37+5)$ \\
\hline
\end{tabular}

\section{References}

1. Danese E, Salvagno GL, Negrini D, Brocco G, Montagnana M, et al (2017) Analytical evaluation of three enzymatic assays for measuring total bile acids in plasma using a fully automated clinical chemistry platform. Plos One 12.

2. Ovadia C, Williamson C (2016) Intrahepatic cholestasis of pregnancy: Recent advances. Clin Dermatol. 34: 327-334.

3. Gudmundsdottir TS, Bjornsson E, Steingrimsdottir T (2018) Intrahepatic Cholestasis of Pregnancy, Epidemiology and Demography over a 10-Year Period in Iceland. Gastroenterology 154: S1207-S1208.

4. Morton A, Laurie J (2018) The biochemical diagnosis of intrahepatic cholestasis of pregnancy. Obstet Med 12: 76-78.

5. Chappell L, Hill R, Conti-Ramsden F, Wade J, Williamson C (2018) Detection of additional abnormalities or co-morbidities in women with intrahepatic cholestasis of pregnancy. Pregnancy Hypertens 13: S49.

6. Ovadia C, Seed PT, Sklavounos A (2019) Association of adverse perinatal outcomes of intrahepatic cholestasis of pregnancy with biochemical markers: results of aggregate and individual patient data meta-analyses. The Lancet 393: 899-909.

7. Yurdakök M (2011) Meconium aspiration syndrome:do we know. Turk J Pediatr 53: 121-129.

8. Salame AA, Jaffal MJ, Mouanness MA, Nasser Eddin AR, Ghulmiyyah LM (2019) Unexplained First Trimester Intrahepatic Cholestasis of Pregnancy: A Case Report and Literature Review. Case Rep Obstet Gynecol 2019: 4980610.

9. Williamson C, Geenes V (2014) Intrahepatic cholestasis of pregnancy. Obstet Gynecol 124: 120-133.

10. Bergman H, Melamed N, Koren G (2013) Pruritus in pregnancy: treatment of dermatoses unique to pregnancy. Can Fam Physician 59: 1290-1294.

11. Ingber A (2009) Pruritus Gravidarum and Intrahepatic Cholestasis of Pregnancy. In: Obstetric Dermatology. Springer, Berlin, Heidelberg 97109.

12. Kokrdová Z, Hůlek P (2008) Intrahepatic cholestasis of pregnancy Intrahepatální cholestáza těhotných. Folia Gastroenterol Hepatol 6: 19-23. 


\section{Global Journal of Reproductive Medicine}

13. Chivers S, Williamson C (2018) Intrahepatic cholestasis of pregnancy Obstet Gynaecol Reprod Med. 28: 215-217.

14. Bacq Y, Sentilhes L (2014) Intrahepatic cholestasis of pregnancy: Diagnosis and management. Clin Liver Dis 4: 58-61.

15. Cummings K, Cottrell, J, Ounpraseuth S (2018) The Effect of Hepatitis C on Maternal Bile Acid Level and the Fetal Left Ventricular Tei Index. Marshall Journal of Medicine 4

16. Xie F, Liu X, Xiao P, Huang Y (2018) Bile Acid Effects on Placental Damage in Intrahepatic Cholestasis of Pregnancy.J Biosci Med 6: 42-52.

17. Brouwers L, Koster MPH, Page Christiaens GCML (2015) Intrahepatic cholestasis of pregnancy: maternal and fetal outcomes associated with elevated bile acid levels. Am J Obstet Gynecol 212(100): e1-e7.

18. Labbe C, Delesalle C, Creveuil C, Dreyfus M (2018) Early and later intrahepatic cholestasis of pregnancy (ICP): Study of adverse pregnancy outcomes. Cholestases intrahepatiques gravidiques (CIG) précoces et tardives : étude des complications materno-fœtales. Gynecol Obstet Fertil Senol 46: 388-394.

19. Lin J, Gu W, Hou Y (2019) Diagnosis and prognosis of early-onset intrahepatic cholestasis of pregnancy: a prospective study. J Matern Fetal Med 32: 997-1003.

20. Cui Y, Xu B, Zhang X, He Y, Shao Y, Ding M (2018) Diagnostic and therapeutic profiles of serum bile acids in women with intrahepatic cholestasis of pregnancy-a pseudo-targeted metabolomics study. Clin Chim Acta 483: 135-141.

21. Chappell LC, Gurung V, Seed PT (2012) Ursodeoxycholic acid versus placebo, and early term delivery versus expectant management, in women with intrahepatic cholestasis of pregnancy: semifactorial randomised clinical trial. BMJ 344: e3799.

22. Paumgartner G, Beuers U (2004) Mechanisms of action and therapeutic efficacy of ursodeoxycholic acid in cholestatic liver disease. Clin Liver Dis 8: 67-81.
23. Paumgartner G, Beuers U (2002) Ursodeoxycholic acid in cholestatic liver disease: Mechanisms of action and therapeutic use revisited. Hepatology 36: 525-531.

24. Shadi AH, Ovadia C, Lieu T (2016) Prognostic and mechanistic potential of progesterone sulfates in intrahepatic cholestasis of pregnancy and pruritus gravidarum. Hepatology 63: 1287-1298.

25. Sinakos E, Lindor KD (2016) Editorial: Bile Acid Profiles in Intrahepatic Cholestasis of Pregnancy: Is This the Solution to the Enigma of Intrahepatic Cholestasis of Pregnancy? Am J Gastroenterol 105: 596598.

26. Feng D, He W (2016) Asymptomatic elevated total serum bile acids representing an unusual form of intrahepatic cholestasis of pregnancy. Int J Gynaecol Obstet 134: 343-344.

27. Adams A, Jacobs K, Vogel RI, Lupo V (2015) Bile Acid Determination after Standardized Glucose Load in Pregnant Women. AJP Rep 5: e168-e171.

28. Martinefski M, Contin M, Lucangioli S, Di Carlo MB, Tripodi V (2012) In search of an accurate evaluation of intrahepatic cholestasis of pregnancy. Scientifica 496-489.

29. Chen J, Deng W, Wang J, Shao Y, Ou M, et al. (2013) Primary bile acids as potential biomarkers for the clinical grading of intrahepatic cholestasis of pregnancy. Int J Gynaecol Obstet 122: 5-8.

30. (2018) Dialab. Bile acids. Enzymatic cycling. Kit's information.

31. Lawrance D, Williamson C, Boutelle MG, Cass AEG (2015) Development of a disposable bile acid biosensor for use in the management of cholestasis. Anal Methods 7: 3714-3719.

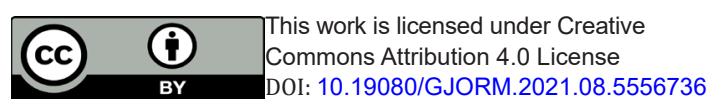

\begin{tabular}{|l|}
\hline \multicolumn{1}{|c|}{ Your next submission with Juniper Publishers } \\
will reach you the below assets \\
- Quality Editorial service \\
- Swift Peer Review \\
- Reprints availability \\
- E-prints Service \\
- Manuscript Podcast for convenient understanding \\
- Global attainment for your research \\
- Manuscript accessibility in different formats \\
( Pdf, E-pub, Full Text, Audio) \\
- Unceasing customer service \\
Track the below URL for one-step submission \\
https://juniperpublishers.com/online-submission.php
\end{tabular}

